\title{
A needs assessment to explore undergraduate medical students' understanding of nutrition during the first one thousand days of life
}

\author{
R.L. Creeden ${ }^{1}$ and C.E. Childs ${ }^{1,2}$ \\ ${ }^{1}$ Faculty of Medicine, University of Southampton, Southampton, UK and \\ ${ }^{2}$ Institute for Life Sciences, University of Southampton, Southampton, UK
}

The first 1,000 days of life are an important period for physiological growth and development ${ }^{(1)}$. Nutritional choices during this time have many health consequences which can influence a lifetime ${ }^{(2)}$. Previous cross sectional, questionnaire-based studies asking medical students focused on only one aspect of nutrition during the first 1,000 days ${ }^{(3)}$. It is important that medical students are familiar with nutrition recommendations so that they feel confident, as future doctors, supporting parents from preconception through childhood and beyond. This needs assessment aimed to explore University of Southampton undergraduate Bachelor of Medicine (BM) medical students' nutrition education needs for the first 1,000 days, by reviewing national and local curricula and using an online survey.

Gap analysis methodology was used to review the current curricula followed by a cross sectional opportunistic online survey to identify what students knew. University of Southampton medical students on either the BM5, BM6 or BMEU undergraduate programmes were eligible to complete the survey and were offered relevant revision notes on completion. The survey contained different question types including multiple choice, ranking, free text responses and Likert scales. Quantitative data analysis was used to identify gaps in understanding.

There were thirty-eight eligible responses to the online survey: 10 males and 28 females. Only $18.4 \%$ of students correctly identified obesity as the most common maternal medical condition. There was no significant association $(p=0.078)$ between the student's year of study and their knowledge of exclusive breastfeeding recommendations. All students were in favour of nutrition topics being included in the BM programme. Only $21.1 \%$ of students correctly identified both Folic Acid and Vitamin D as the recommended supplements during pregnancy and 5.3\% justified why. $23.7 \%$ of students incorrectly thought Vitamin B12 was recommended for all pregnant women. Around half of students (55.3\%) could correctly recommend exclusive breastfeeding for a duration of 6 months and the majority of those who were incorrect suggested a longer rather that shorter duration of breastfeeding $(34.2 \%)$. $84 \%$ of students recalled the immune benefits of breastfeeding. $52.6 \%$ of students identified pain or discomfort as reasons why women may not breastfeed. Mothers' experiences of feeding with a previous child were considered most likely to influence their feeding choices $(55.3 \%)$.

This Needs Assessment aimed to understand University of Southampton medical students' knowledge, attitudes and confidence regarding nutrition during the first 1,000 days of life. Through a curriculum appraisal, taught content was established, and student knowledge and confidence ascertained using an online survey. The results will inform development of appropriate resources to target knowledge gaps regarding nutrition during pregnancy, breastfeeding and weaning. It is important that medical students are competent in infant nutrition skills and counselling for their roles as future doctors.

\section{References}

1. Osmond C \& Barker DJ (2000) Environ Health Perspect 108, 545-553.

2. Baidal JAW, Locks LM, Cheng ER et al. (2016) Am J Prev Med 50, 761-779.

3. Biggs KV, Fidler KJ, Shenker NS et al. (2020) Int Breastfeed J 15, 46. 